\title{
Temperature sensitivity of silica micro-resonators
}

\author{
Qiulin Ma, Tobias Rossmann and Zhixiong Guo ${ }^{1}$ \\ Department of Mechanical and Aerospace Engineering, Rutgers, The State University of New Jersey, \\ Piscataway, NJ 08854, USA \\ E-mail: guo@jove.rutgers.edu
}

Received 1 August 2008, in final form 3 August 2008

Published 3 December 2008

Online at stacks.iop.org/JPhysD/41/245111

\begin{abstract}
Optical resonance shifts are measured against temperature changes for different silica beads ranging from 80 to $450 \mu \mathrm{m}$ in diameter. A micro-bead is fabricated by hydrogen flame fusing the tip of a single mode silica fibre/taper, and coupled to a fibre taper of submicrometre diameter. The coupling system in whispering-gallery modes is placed in an insulated cell. The air in the cell is slowly heated up from room temperature to about $10 \mathrm{~K}$ higher, and red shifts of a resonance wavelength during the heating process are recorded. Linear dependence of the wavelength shift versus the temperature rise is observed for all the tested micro-resonators. The measured sensitivity for beads greater than $200 \mu \mathrm{m}$ in size closely matches the analytical value based on bulk material properties of silica thermal expansion and the thermo-optic effect. For smaller micro-beads, however, the measured sensitivity increases with shrinking bead size. The ultra-high resolution of such a kind of temperature micro-sensor and its potential applications are addressed.
\end{abstract}

(Some figures in this article are in colour only in the electronic version)

\section{Introduction}

Optical whispering-gallery modes (WGM) in dielectric microresonators have attracted considerable attention over the past decade in a broad research field from experimental cavity quantum electrodynamics [1], frequency stabilization [2], microlasers $[3,4]$ to micro-sensors $[5,6]$, etc, due to their distinct feature of a very high quality $(Q)$ factor in a small mode volume [7]. A mode propagates on a circular path around a micro-resonator which can be a sphere [7], a disc [8], a ring [9], or a torus [10] corresponding to electromagnetic waves of a specific wavelength trapped and internally reflected in an orbit within the resonator surface of any circular shape.

WGM resonance frequencies depend on the size and refractive index of a resonator. A small change in size and/or refractive index can cause a significant resonance frequency shift for a given mode. This feature is broadly explored for use in WGM-based sensors [5,6]. On the other hand, the material properties of a resonator are susceptible to thermal fluctuations caused by either the ambient temperature variation or the absorption of laser energy during laser scanning or

\footnotetext{
1 Author to whom any correspondence should be addressed.
}

pumping. Thermal expansion and thermo-optic effect are two noticeable material properties, impacting the resonator size and refractive index, respectively. Cai et al [11] found red shifts on the frequency of a single mode in a glass microchip laser when the pump power is increased and quantitatively explained the phenomenon based on the thermal expansion effect of the chip material. As stability and continuous operation are critical for use of a micro-resonator, Carmen et al [12] demonstrated a self-stable thermal equilibrium solution for the micro-cavity-pump system. Recently, Han and Wang [13] proposed qualitatively the use of a surface layer with a negative thermo-optic coefficient to compensate the thermal drift of a resonance frequency in an optical micro-resonator.

In this study, we investigate the resonance wavelength shifts of fused silica micro-beads with well-controlled temperature changes. Various micro-beads about 50-500 $\mu \mathrm{m}$ in diameter were fabricated by fusing the tip of a single mode fibre or a fibre taper. A fibre taper of submicrometre waist size was pulled and used for phase-matched coupling [14]. To accurately characterize the sensitivity (WGM shift versus temperature change), the taper-bead coupling system is placed in a thermally insulated cell in which the temperature is slowly and steadily heated up from room temperature to about $10 \mathrm{~K}$ 


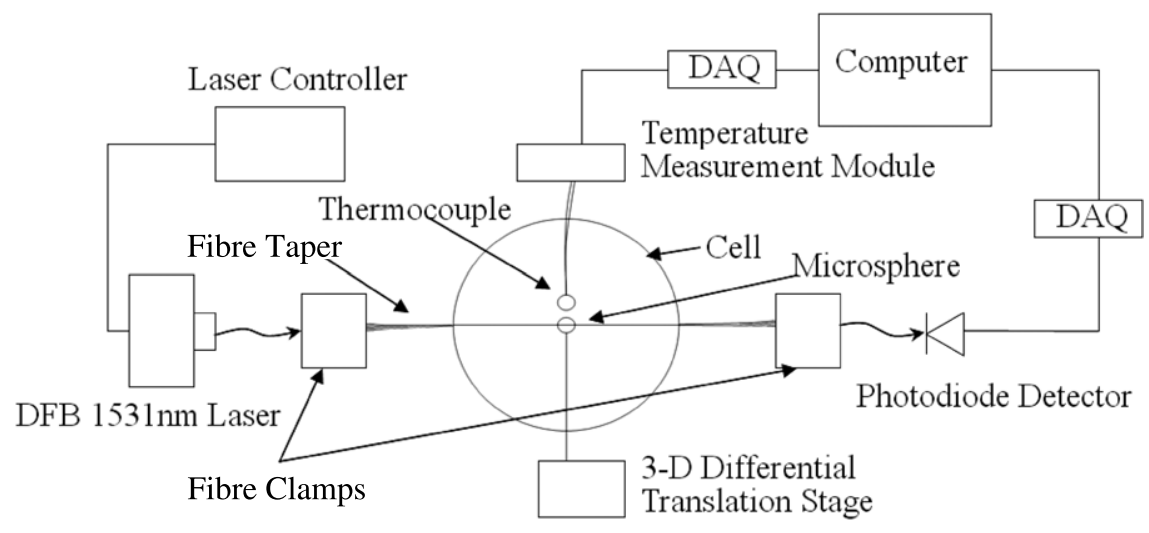

Figure 1. Diagram of the experimental setup.

higher with little convection effect. The frequency shifts of a resonance mode are then recorded for every 0.5 or $1 \mathrm{~K}$ air temperature increment in the cell. The thermal equilibrium and response times of the micro-beads are analysed. The temperature curves of several tested micro-beads of different sizes are plotted. The experimental data are treated with least-squares linear fitting and the measured temperature sensitivities of the micro-beads are obtained. The theoretical temperature sensitivities of the micro-beads are analysed and calculated based on the bulk silica material properties of thermal expansion and the thermo-optic effect. Comparisons between the analyses and the experimental measurements are made. The limitation of bulk material properties is discussed. The ultra-high temperature resolution of WGM systems is analysed. Potentials of WGM-based temperature microsensors are discussed.

\section{Experimental setup}

Figure 1 shows the diagram of our experimental setup. The micro-bead is precisely positioned by a 3D differential translation stage (Thorlabs) to couple with the fibre taper in the cell. The fibre taper is held by two fibre clamps. The microbead-taper coupling is observed under a stereo-microscope (Zeiss SV8) to place the micro-beads in contact with the taper. Optimum coupling between a micro-bead and a fibre taper is a very complicated problem [7,14], depending on the size compatibility of the micro-bead, taper and gap [15]. A DFB $1531 \mathrm{~nm}$ laser (NEL NLK1556STG) is tuned and launched into the fibre taper. The transmission signal is detected by a photodiode detector (Thorlabs PDA400) and recorded by a computer equipped with DAQ cards (National Instrument). A thermocouple (Omega K-type with $0.01^{\prime \prime}$ bead and $0.4 \mathrm{~s}$ time constant) in the cell is positioned about $0.2 \mathrm{~mm}$ away from the micro-bead to monitor the temperature change around the micro-bead. The temperature measured by the thermocouple is recorded simultaneously with the recording of the WGM resonance spectrum with a temperature measurement module (National Instrument).

Figure 2 shows a photograph of the working cell which is made of a plastic tube $1^{\prime \prime}$ in diameter and $2^{\prime \prime}$ in length. The accesses on the tube side for placing the fibre taper,

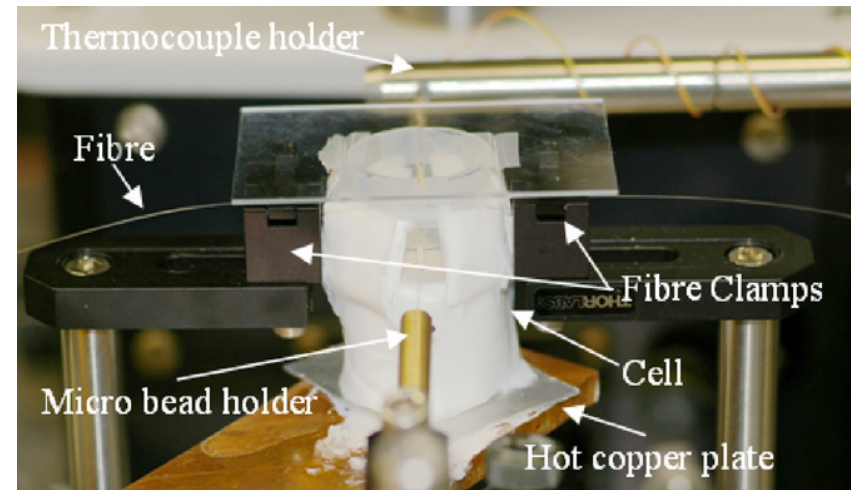

Figure 2. Photograph of the working cell.

thermocouple and micro-bead are sealed. The tube is wrapped with layers of delicate task wipers (Kimwipes) for thermal insulation. The top of the cell is covered by a transparent glass slide, avoiding environmental disturbance from the room to the cell. The bottom of the cell is a $2 \mathrm{~mm}$ thick aluminium plate which is glued to a copper plate with silicon paste to ensure excellent heat conduction. The copper plate is slowly heated up from room temperature. It takes about $3 \mathrm{~min}$ to steadily raise the air temperature in the cell monitored by the thermocouple by $1 \mathrm{~K}$. The air inside the cell is visually stagnant during the heating process with minimized heat convection effect. Uniform temperature increment and distribution in the micro-bead are important.

\section{Preparation of micro-beads and tapers}

The key components of the WGM temperature sensor are the micro-bead as a resonator and the fibre taper as a nearfield coupler. In our study, micro-beads about 50-500 $\mu \mathrm{m}$ in diameter are fabricated by oxy-hydrogen flame (about $2800-3080 \mathrm{~K}$ ) fusing the end of a very clean single mode fibre (Corning SMF-28) or fibre taper. This technique has been found to produce the minimal amount of soot that can spoil the quality of micro-beads [7]. Two typical micro-beads that we fabricated are shown in figure $3(a)$.

The fibre taper is fabricated through the heating and pulling technique. One end of a stripped single mode fibre 


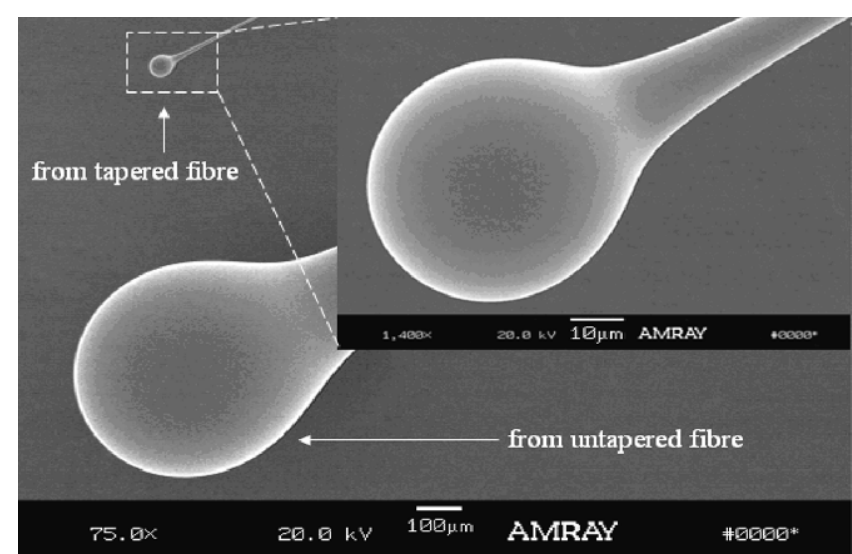

(a)

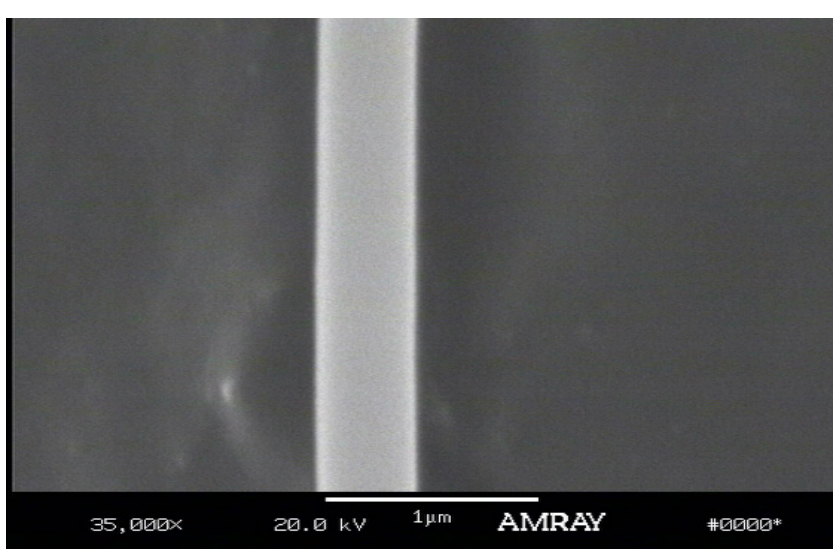

(b)

Figure 3. (a) SEM image of two typical micro-beads fabricated from an untapered fibre tip and a fibre taper, respectively, and (b) SEM image of the waist of a fibre taper.

(Corning SMF-28) is fixed and the other end is pulled with a computerized stepper motor. During the pulling procedure, an oxy-hydrogen flame (about $2400 \mathrm{~K}$ ) is used for heating and softening the taper waist. The DFB laser delivers continuous wave light of stabilized intensity to the fibre during the fabrication of a fibre taper and its transmission signal through the taper is dynamically monitored. We have achieved negligible transmission loss during the tapering. The waist size of a taper can be measured by a scanning electron microscope (SEM) and the SEM image of a typical taper we made is shown in figure $3(b)$. Clearly the taper size is in the submicrometre level. To preserve the propagation of a fundamental mode light in a tapered region, the adiabatic condition [16] should be examined. For our DFB $1531 \mathrm{~nm}$ tunable laser and light delivery SMF-28 fibre, it is calculated that the transitional tapered region must be longer than $2.51 \mathrm{~mm}$ to satisfy this adiabatic condition. All the tapers fabricated for this study are satisfactory.

\section{Results and discussion}

The need for an insulated working cell in the experiments is justified below. To test the influence of open air on the microbead, we measured the dynamic shifts of a WGM resonance wavelength over $10 \mathrm{~s}$ for a $105 \mu \mathrm{m}$ diameter bead suspended

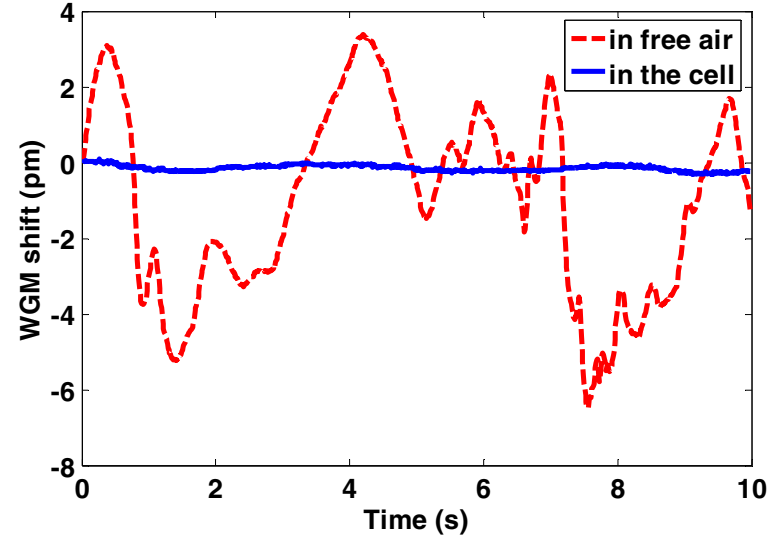

Figure 4. The measured dynamic wavelength shifts of a WGM resonance of the micro-bead of $105 \mu \mathrm{m}$ diameter placed in free air and in the working cell at $298 \mathrm{~K}$, respectively.

in free air of the lab room and in the working cell (at $298 \mathrm{~K}$ ), respectively. The results of these two cases are compared in figure 4. It is observed that the wavelength shift fluctuates with an amplitude of $5 \mathrm{pm}$ for the case when the sphere is placed in free air; while the fluctuation amplitude is less than $0.1 \mathrm{pm}$ for the case when the sphere is placed inside the cell. A reduction of 50 times in fluctuation is achieved when the working cell is utilized. From the later result in table 1 , a shift of $0.1 \mathrm{pm}$ in the WGM sensor corresponds to a temperature variation of about $0.007 \mathrm{~K}$. A fluctuation of $5 \mathrm{pm}$ in the case of open air results in a temperature error of $0.35 \mathrm{~K}$. Thus, the use of the working cell effectively protects the WGM coupling system from ambient room disturbances (such as air convection and room temperature fluctuation) and improves significantly the temperature stability around the coupling system.

In order to let the micro-bead, the thermocouple and the surrounding air be in thermal equilibrium during a measurement, the air in the cell has to be stable and the air temperature must be increased very slowly and steadily. The thermal response time of the silica micro-beads can be estimated using the lumped capacity analysis of heat transfer [17]. For example, the time constant of a silica micro-bead $450 \mu \mathrm{m}$ in size is about $0.9 \mathrm{~s}$, and it takes about $4.5 \mathrm{~s}$ for the whole bead to reach thermal equilibrium. The smaller the bead, the shorter the thermal response time. The thermal time constant of a micro-bead $80 \mu \mathrm{m}$ in size is only $0.03 \mathrm{~s}$. In actual experiments, the copper plate is heated so that it takes about $3 \mathrm{~min}$ to raise the air temperature by $1 \mathrm{~K}$. For every 0.5 or $1 \mathrm{~K}$ temperature increment, $10 \mathrm{WGM}$ spectra are measured in $0.1 \mathrm{~s}$ and an averaged resonance wavelength shift is calculated. We are confident that the WGM couple and thermocouple are in thermal equilibrium and have a nearly constant and uniform temperature distribution during the samplings.

Now we are ready to investigate the resonance wavelength shift against the temperature rise. The wavelength tuning range for each measurement is measured by an optical spectrum analyzer (ANDO AQ6317B). A significant WGM resonance that appears in each tuning is selected for measuring the resonance wavelength shift. A WGM spectrum at room temperature is first measured. Then a wavelength shift against 
Table 1. Temperature sensitivities of the tested micro-beads.

\begin{tabular}{lllll}
\hline $\begin{array}{l}\text { Micro-bead } \\
\text { diameter }(\mu \mathrm{m})\end{array}$ & $\begin{array}{l}\text { Resonance wavelength at } \\
\text { room temperature }(\mathrm{nm})\end{array}$ & $\begin{array}{l}\text { Analytical sensitivity } \\
\left(\mathrm{pm} \mathrm{K}^{-1}\right)\end{array}$ & $\begin{array}{l}\text { Measured sensitivity } \\
\left(\mathrm{pm} \mathrm{K}^{-1}\right)\end{array}$ & $\begin{array}{l}\text { Relevant coefficient } \\
\text { in linear fitting }\end{array}$ \\
\hline 83 & 1531.575 & 13.89 & $18.31 \pm 0.17$ & 0.9996 \\
108 & 1530.337 & 13.88 & $16.63 \pm 0.20$ & 0.9992 \\
145 & 1531.978 & 13.90 & $15.39 \pm 0.11$ & 0.9997 \\
210 & 1531.954 & 13.90 & $13.83 \pm 0.16$ & 0.9993 \\
290 & 1531.113 & 13.89 & $13.98 \pm 0.16$ & 0.9993 \\
430 & 1530.335 & 13.88 & $14.12 \pm 0.22$ & 0.9986 \\
\hline
\end{tabular}
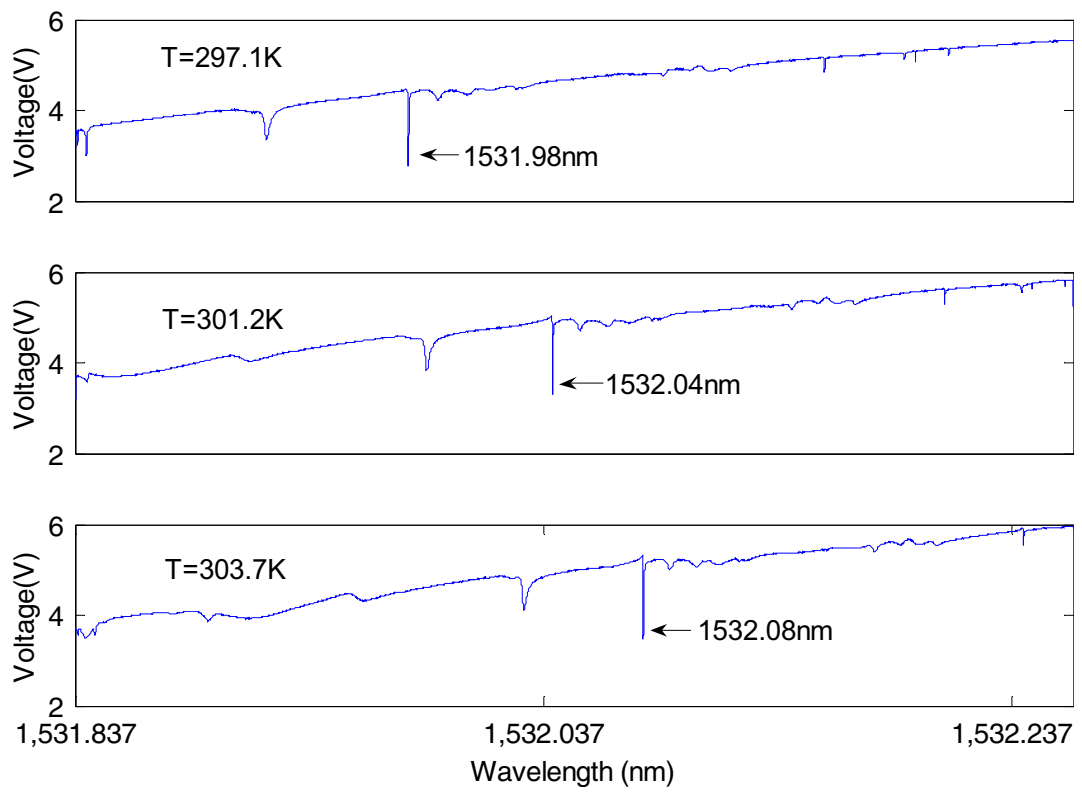

Figure 5. The measured transmission spectra of the micro-bead of $145 \mu \mathrm{m}$ diameter at three different temperature stages.

a temperature rise is obtained from the difference between the resonance wavelength at any measured temperature and that at room temperature. Figure 5 shows the measured WGM spectra for the micro-bead $145 \mu \mathrm{m}$ in diameter at three different temperature stages. The loaded $Q$ value of the micro-bead is estimated in a range from $8 \times 10^{6}$ to $4 \times 10^{7}$ from the dips indicated in the figure.

Micro-beads with diameters of $83 \mu \mathrm{m}, 108 \mu \mathrm{m}, 145 \mu \mathrm{m}$, $225 \mu \mathrm{m}, 290 \mu \mathrm{m}$ and $430 \mu \mathrm{m}$, respectively, are measured for wavelength shifts versus temperature changes in a general temperature range from room temperature to about 5-10 K higher and the results are presented in figure 6 . The temperature sensitivity (wavelength shift per unit temperature change) for each of the tested micro beads can be best determined by linear fitting of the experimental data. The measured sensitivity results as well as the analytical ones are listed and compared in table 1.

Assuming a WGM circulates as a $k$ th polygon in a microresonator and the resonance in an azimuthal mode $m$, it follows that $k D \sin \pi / k=m \lambda / n$, where $\lambda$ is the resonance wavelength in vacuum and $D$ and $n$ are the diameter and refractive index of the resonator, respectively. Taking a variation, we have $\mathrm{d} n / n+\mathrm{d} D / D=\mathrm{d} \lambda / \lambda$. Assume linear thermal expansion and thermo-optic effects [18]: $\mathrm{d} D / D=\alpha \mathrm{d} T$ and $\mathrm{d} n / n=$ $\beta \mathrm{d} T$, where $\alpha$ and $\beta$ are the thermal expansion coefficient and thermo-optic coefficient, respectively. The temperature

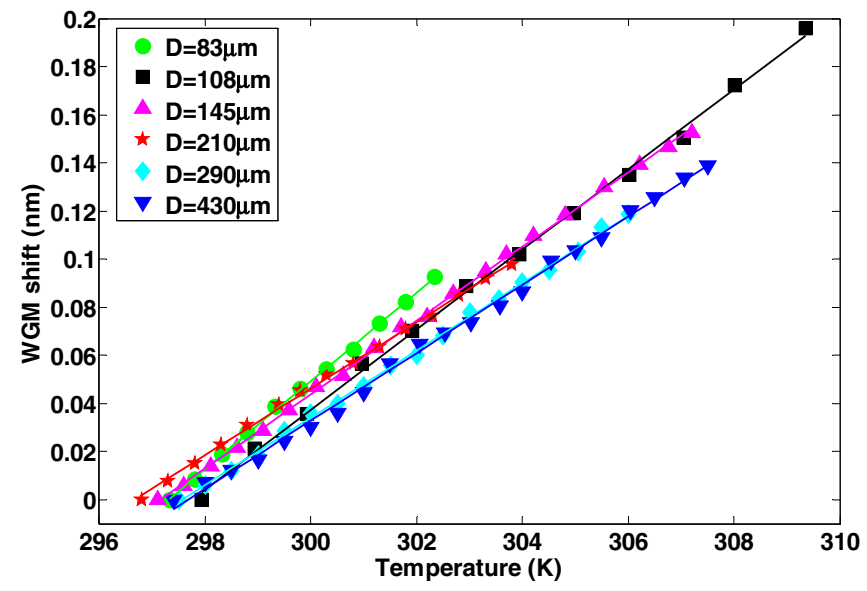

Figure 6. The measured data and linear fitting curves of WGM resonance wavelength shifts against temperature variation for the tested micro-beads.

sensitivity of a micro-bead is analysed as $\mathrm{d} \lambda / \mathrm{d} T=(\alpha+\beta) \lambda$. Since the magnitude of a shift is much smaller than a resonance wavelength in the testing temperature range, we can use the measured resonance wavelength at a reference temperature (such as room temperature) to calculate the temperature sensitivity for a micro-bead operated at a given resonance mode. The analytical temperature sensitivities in table 1 
Table 2. Measured silica property values of various micro-beads.

\begin{tabular}{lllllll}
\hline $\begin{array}{l}\text { Micro-bead } \\
\text { diameter }(\mu \mathrm{m})\end{array}$ & 83 & 108 & 145 & 210 & 290 & 430 \\
\hline$\alpha+\beta\left(10^{-6} \mathrm{~K}^{-1}\right)$ & $11.96 \pm 0.11$ & $10.87 \pm 0.13$ & $10.05 \pm 0.07$ & $9.03 \pm 0.10$ & $9.13 \pm 0.10$ & $9.23 \pm 0.14$ \\
\hline
\end{tabular}

are calculated using the resonance wavelengths of the microbeads measured at room temperature and the values of $\alpha=$ $0.55 \times 10^{-6} \mathrm{~K}^{-1}[19]$ and $\beta=8.52 \times 10^{-6} \mathrm{~K}^{-1}$ [20] for bulk silica at a wavelength of around $1531 \mathrm{~nm}$ and at a temperature of about $303 \mathrm{~K}$. Since a resonance wavelength depends on the size of a resonator, slight differences in the sensitivities among the different beads are observed. Nevertheless, the variations are so small $\left( \pm 0.01 \mathrm{pm} \mathrm{K}^{-1}\right)$ that the analytical temperature sensitivity for all the tested micro-beads is nearly a constant at the average value of $13.89 \mathrm{pm} \mathrm{K}^{-1}$.

From table 1, it is seen that the relevant coefficients in the least-squares linear fittings of the experimentally measured data are all close to 0.999 which indicates an excellent linear dependence of the resonance wavelength shift on the temperature change. This is consistent with the fact that theoretically the temperature sensitivity is nearly a constant. It is seen from table 1 that for micro-beads with diameter above $200 \mu \mathrm{m}$ the sensitivity difference between the analytical and experimental results is within $1.5 \%$. The measurements are in excellent agreement with the analysis. Both the geometric optics interpretation of the WGM resonance and the use of the $\alpha$ and $\beta$ values from bulk silica material in the sensitivity analysis are successful in the case of large beads $(>200 \mu \mathrm{m})$.

Noticeably the measured temperature sensitivities from the experiments do not match with the analytical prediction for micro-beads with the diameter below $150 \mu \mathrm{m}$. The measured value is bigger than the analytical one and increases with reducing bead size. The smaller the micro-bead, the more sensitive it is. For example, the measured sensitivity is about $32 \%$ more than the analytical value for the micro-bead of $83 \mu \mathrm{m}$ diameter. Since the equatorial circumference of this sphere is still much longer than the resonance wavelength, the geometric optics interpretation of the WGM resonance in the analysis of temperature sensitivity should still be valid. The problem should be with the use of the two values $\alpha$ and $\beta$ in the analysis.

The $\alpha$ and $\beta$ values used for calculating the analytical sensitivity are based on the properties of bulk silica material. When the resonator is shrunk to the micrometre scale, our measurements indicate that the sum of $\alpha$ and $\beta$ coefficients of silica increases with the reduction in material size (see table 2). This finding is new and it requires new knowledge in micro/nano-scale material sciences. The data in table 2 were inversely obtained from the measured sensor sensitivity values in table 1 . The tolerances were the standard deviations from the respective curve data in figure 6. Further measurements for thermal expansion and thermo-optic effect coefficients in micro/nano-scale materials are needed.

Since the linearity of the experimental data curves in figure 6 for the small micro-beads $(<150 \mu \mathrm{m})$ is excellent, the sum of $\alpha$ and $\beta$ coefficients should be a very weak function of temperature in the testing temperature range. Thus, a resonator with size in the micrometre level can still be developed as a temperature micro-sensor. In addition, a WGM sensor with the resonator size down to the micrometre level has even larger sensitivity. The extraordinary demand for integrated techniques and miniaturization presents a major challenge to the sensor community.

WGM-based temperature sensors are basically frequency modulated (FM) sensors because the resonance frequency is modulated. A FM sensor is normally more sensitive, or in other words, has much finer measurement resolution than an intensity modulated (IM) sensor such as a thermocouple. Pico Technology did a press announcement in 2000 that TC-08 thermocouple converters boast of $2.5 \times 10^{-2}$ resolution. Recently, Jha et al [21] achieved a high resolution of approximately $8 \times 10^{-3} \mathrm{~K}$ using a complementary metal oxide semiconductor (CMOS) digital temperature sensor. Conventional CMOS devices work over a range of $-55^{\circ} \mathrm{C}$ to $+125^{\circ} \mathrm{C}$. Thermocouples are most suitable for measuring over a large temperature range from $-273^{\circ} \mathrm{C}$ up to $1800^{\circ} \mathrm{C}$. The temperature range of a WGM-based sensor depends on the material of the micro-resonator. Silica material still functions below $-233^{\circ} \mathrm{C}$ and its softening point is as high as $1665^{\circ} \mathrm{C}$.

The temperature resolution of the WGM sensor depends on two factors: the wavelength/frequency resolution of the instrument and the temperature sensitivity of the transducer (i.e. the silica micro-bead). Suppose the instrument can resolve the wavelength at a resolution of $\Delta \lambda_{\min }$, the temperature measurement resolution of a WGM-based sensor is then obtained as

$$
\Delta T_{\min }=\Delta \lambda_{\min } /(\mathrm{d} \lambda / \mathrm{d} T) .
$$

The wavelength resolution is the largest one among the linewidth of the scanning laser, the resolution of the data acquisition and processing system and one-tenth of the WGM resonance linewidth. Suppose the wavelength tuning range of a scanning laser is $\Delta \lambda$, the tuning frequency of the laser is $f_{\mathrm{s}}$ and the DAQ card has a sampling rate $S$, then the minimum tuning wavelength is $\Delta \lambda f_{\mathrm{s}} / S$.

In the current instrument, the DAQ card has a sampling rate of $2.5 \mathrm{MHz}$. The tuning wavelength range of the DFB laser is between 0.03 to $1 \mathrm{~nm}$. We actually tuned the laser in a frequency of $100 \mathrm{~Hz}$ with a tuning range of $0.40 \mathrm{~nm}$ for the $145 \mu \mathrm{m}$ diameter sensor. Thus, the wavelength resolution for the data acquisition system is $1.60 \times 10^{-5} \mathrm{~nm}$ which is larger than the factory-specified linewidth of the current laser ( $2 \mathrm{MHz}$, i.e. $\left.1.56 \times 10^{-5} \mathrm{~nm}\right)$. One-tenth of a resonance linewidth $(\lambda / Q)$ is $1.53 \times 10^{-5} \mathrm{~nm}$ if we use a loaded $Q$ value of around $10^{7}$ for the calculation. Considering its measured sensitivity $0.0154 \mathrm{~nm} \mathrm{~K}^{-1}$ in table 1 , the temperature resolution of this WGM sensor in our experiment is then calculated as $1.0 \times 10^{-3} \mathrm{~K}$. This value is one order of magnitude smaller 
than the resolution of a high resolution thermocouple and is also much finer than the resolution of the CMOS sensor cited above.

The temperature resolution of WGM-based sensors may further be improved by reducing the data acquisition resolution, using a narrow linewidth laser, increasing the loaded $Q$ value and increasing the sensor sensitivity. The stateof-the-art lasers currently can push to a linewidth as narrow as a few kilohertz, i.e. almost three orders of magnitude reduction as compared with the laser in the current instrument. The data sampling resolution can also go down to this order. $Q$ values up to $10^{10}$ were also reported in the literature. Hence, it is possible to detect a minor temperature change to the level of $10^{-6} \mathrm{~K}$.

In addition to ultra-high resolution, WGM micro-sensors have other promising application potentials. For example, on-chip temperature monitoring is currently a challenging task [22] because a chip may be easily overheated to function incorrectly or sustain fatal damage without proper temperature monitoring and thermal solution. Nevertheless, integrating common types of sensors (such as thermodiodes, thermistors or thermocouples) into a chip is technically difficult. However, WGM sensors can be easily fabricated and integrated into other electro-optical systems using existing microelectronics techniques [23]; furthermore, the thermo-optic effect can be dominant as in the silica resonators $(\beta \gg \alpha)$ and this will minimize the mechanical stress induced to the chip by thermal expansion. Recent developments in micro-fluidics and lab-on-a-chip devices have also raised the challenge of whole-chip (liquid and substrate) temperature measurement [24]. Traditional embedded thermocouples have been used for micro-fluidics applications. However, measurements are limited to single locations unless complex fabrication and data acquisition are implemented for multiple thermocouples $[25,26]$. It is possible that arrays of WGM sensors can be fabricated on a chip and coupled with just one waveguide to monitor temperature at different locations.

In summary, micro-bead-taper coupling systems are fabricated and built in a working cell which is disturbance free and has a well-controlled temperature change. WGM spectra of the coupling systems in a temperature range from room temperature to $5-10 \mathrm{~K}$ above room temperature are measured. Red shifts of a resonance mode against the temperature rise are recorded. Beads with size from micrometre to sub-millimetre are tested. Excellent linear dependence of the resonance wavelength shift versus the temperature change is observed for all the tested sensors. A good agreement of temperature sensitivity is found between the measurement and the analysis for micro-beads of diameter greater than $200 \mu \mathrm{m}$. However, the measured sensitivity of smaller micro-beads $(<150 \mu \mathrm{m})$ is different from the analytical prediction which is deduced from the bulk silica properties. Though the thermal drift phenomenon has been discovered before, enlarged thermal drift with shrinking resonator size in the micrometre scale is found for the first time. The temperature resolution of the WGM system is found to be extremely high. The improved sensitivity, miniaturization, integration and ultra-high resolution enable broad application potentials of WGM-based sensors.

\section{Acknowledgments}

Support of the Academic Excellence Funds Award from Rutgers University to this project is gratefully acknowledged. Part of this material is also based upon the work supported by the National Science Foundation under Grant No CBET0651737.

\section{References}

[1] Vernooy D, Furusawa A, Georgiades N, Ilchenko V and Kimble H 1998 Phys. Rev. A 572293

[2] Vassiliev V, Velichansky V, Ilchenko V, Gorodetsky M, Hollberg L and Yarovitsky A 1998 Opt. Commun. 158182

[3] Sandoghdar V, Treussart F, Hare J, Lefevre-Seguin V, Raimond J and Haroche S 1996 Phys. Rev. A 541777

[4] Cai M, Painter O, Vahala K and Sercel P 2000 Opt. Lett. 251430

[5] Arnold S, Khoshsima M, Teraoka I, Holler S and Vollmer F 2003 Opt. Lett. 28272

[6] Quan H and Guo Z 2005 J. Quant. Spectrosc. Radiat. Transfer 93231

[7] Gorodetsky M, Savchenkov A and Ilchenko V 1996 Opt. Lett. 21453

[8] Quan H and Guo Z 2007 Nanotechnology 18375702

[9] Sumetsky M, Windeler R, Dulashko Y and Fan X 2007 Opt. Express 1514376

[10] Ilchenko V, Gorodetsky M, Yao X and Maleki L 2001 Opt. Lett. 26256

[11] Cai Z, Chardon A, Xu H, Feron P and Michel Stephan G 2002 Opt. Commun. 203301

[12] Carmon T, Yang L and Vahala K 2004 Opt. Express 124742

[13] Han M and Wang A 2007 Opt. Lett. 321800

[14] Knight J, Cheung G, Jacques F and Birks T 1997 Opt. Lett. 221129

[15] Guo Z, Quan H and Pau S 2006 J. Phys. D: Appl. Phys. 395133

[16] Synder A and Love J 1983 Optical Waveguide Theory (London: Chapman and Hall) p 409

[17] Incropera F and Dewitt D 1996 Introduction to Heat Transfer (New York: Wiley) p 468

[18] Ghosh G 1998 Handbook of Thermo-Optic Coefficients of Optical Materials with Applications (San Diego, CA: Academic) p 122

[19] http://accuratus.com/fused.html

[20] Leviton D and Frey B 2006 Proc. SPIE 627362732

[21] Jha C M, Bahl G, Melamud R, Chandorkar S A, Hopcroft M A, Kim B, Agarwal M, Salvia J, Mehta H and Kenny T W 2007 Appl. Phys. Lett. 91074101

[22] Shih Y and Hwu J 2001 IEEE Electron Device Lett. 22299

[23] Guo Z, Quan H and Pau S 2006 Appl. Opt. 45611

[24] Samy R, Glawdel T and Ren C 2008 Anal. Chem. 80369

[25] Ross D and Locascio L 2003 Anal. Chem. 751218

[26] Erickson D, Liu X, Venditti R, Li D and Krull U 2005 Anal. Chem. 774000 